\title{
Primary Intracranial Leiomyosarcoma - Case Report and Principles for Treatment
}

\author{
Homajoun Maslehaty', Arya Nabavi ${ }^{2}$, Hubertus Maximilian Mehdorn ${ }^{2}$ \\ 'Department of Neurosurgery, University Hospitals Essen, Germany \\ ${ }^{2}$ Department of Neurosurgery, University Hospitals Schleswig-Holstein, Kiel, Germany
}

Article Info

\section{Article Notes}

Received: April 11, 2016

Accepted: July 08, 2016

\section{${ }^{*}$ Correspondence:}

Dr. Homajoun Maslehaty,

Department of Neurosurgery,

University Hospitals Essen, Germany,

Hufelandstrasse 55 ,

45122 Essen, Germany

Telephone: +49 2017230

Email: h.maslehaty@gmx.de

(C) 2016 Maslehaty $H$. This article is distributed under the terms of the Creative Commons Attribution 4.0 International License

\section{ABSTRACT}

Introduction: Primary intracranial leiomysarcoma (LMS) is a rare clinical entity. We present a case of a patient with primary intracranial LMS ten years after surgical resection of a pilocytic astrocytoma in the posterior fossa and highlight different diagnostic and therapeutical features.

\section{Methods: Case report}

Results: A 43 years old male presented with an indolent swelling at occiput without neurological symptoms. Ten years earlier he had undergone surgery elsewhere for an astrocytoma WHO grade 1 of the right cerebellar hemisphere, without following radiotherapy. MRI showed a contrast enhancing lesion of the right posterior fossa infiltrating the occipital bone. Proton magnetic resonance spectroscopy (MRS) indicated malignancy. The tumor could be removed totally with retention of the transverse sinus with negative surgical margins. Histological work-up revealed LMS grade 2. Staging examinations with CT of the chest and abdomen revealed no other tumor. Three months later the patient presented again with a right sided swelling of the neck. MRI of the neck showed contrast enhancing space occupying tissue bordering the former operations field. Surgery was done in view of a recurrent tumor, however, histological work up showed scar tissue without tumor. Nevertheless the patient received GD 192iridium - HDR - brachytherapy up to 20 Gy and radiotherapy of the corresponding muscle tissue with 50Gy. Three and six months follow up MRI of the brain and spine showed no recurrence.

Conclusion: Primary LMS of the CNS is very infrequent. Reported cases of intracranial LMS are often metastasic. An elevated incidence in association with Ebstein-Barr virus, AIDS and after radiotherapy is described. Neuro-imaging should contain MRI, whereby MRS is helpful to identify malignancy. In view of longest survival time, a complete tumor resection with negative margins is to be aspired. After diagnosis of LMS, adjuvant radiotherapy is recommended, because $40-50 \%$ of soft tissue sarcomas show remission. Success of adjuvant chemotherapy is currently uncertain. Despite multimodal aggressive therapy, prognosis of LMS remains poor, because of early haematogenous spread.

\section{Introduction}

Leiomyosarcoma (LMS) is a malignant neoplasm of smooth muscle cells usually with an aggressive growth tendency. Because of ubiquitous appearance of smooth muscle cells, every organ can be affected. Involvement of the central nervous system (CNS) is a very rare clinical entity; most arises with dural attachment ${ }^{1-3}$. Cases with spread of LMS into the CNS are reported infrequently ${ }^{1,4}$. We present a case of a 43 year old male patient with de novo occurrence of intracranial LMS, review the literature and describe principles for a multimodal therapy. 


\section{Case Presentation}

Tenyears afterneurosurgical resection ofanastrocytoma WHO grade 1 of the right cerebellar hemisphere, without following radiotherapy, a 43 year old male patient presented with a right sided rapid growing indolent palpable swelling at the occiput without neurological symptoms. Cranial MRI showed a contrast enhancing dural based extra axial lesion of the right posterior fossa infiltrating the occipital bone but supposedly sparing the brain parenchyma (Figure 1). The lesion was thought to be of dural origin (i.e. meningioma or hemangiopericytoma), but recurrent astrocytoma following malignant transformation could not be excluded. On cerebral angiography, tumor feeding vessels of the right vertebral, right posterior cerebral and middle meningeal arteries were apparent (not shown). The right transverse sinus was patent. An increased choline (CHO) / creatine (CR) ratio on proton magnetic resonance spectroscopy (MRS) indicated malignancy. Because the neuronal marker $\mathrm{N}$-acetylaspartate was reduced or absent (Figure 2), the tumor was unlikely to be a primary brain tumor.

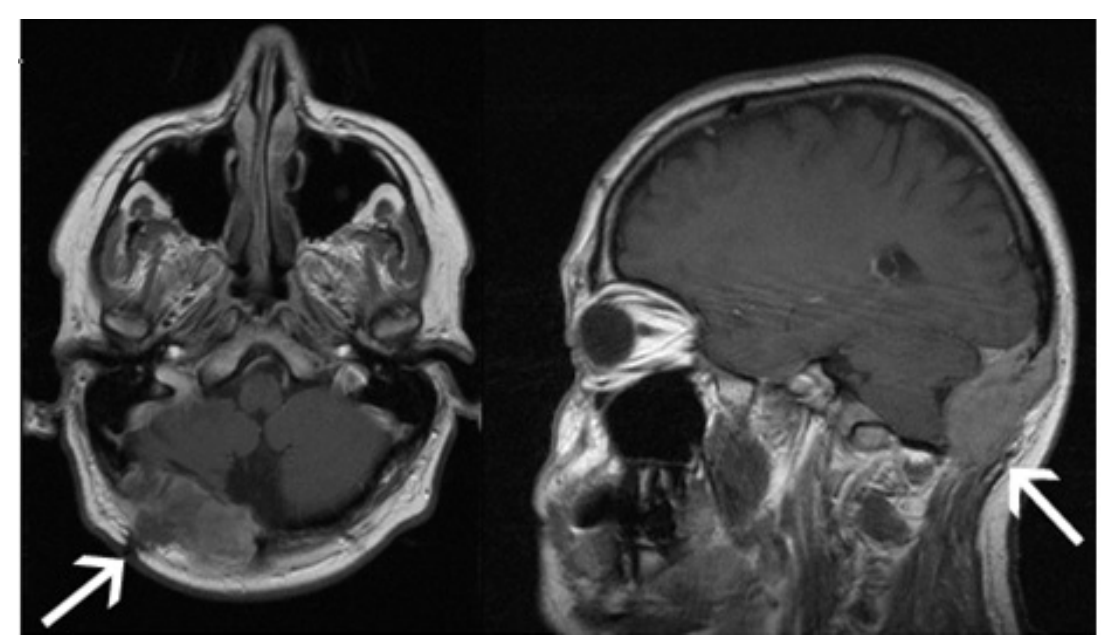

Figure 1: T1 weighted cranial MRI (left axial, right sagittal) demonstrates homogeneous contrast enhancement of the posterior fossa tumor (arrows).

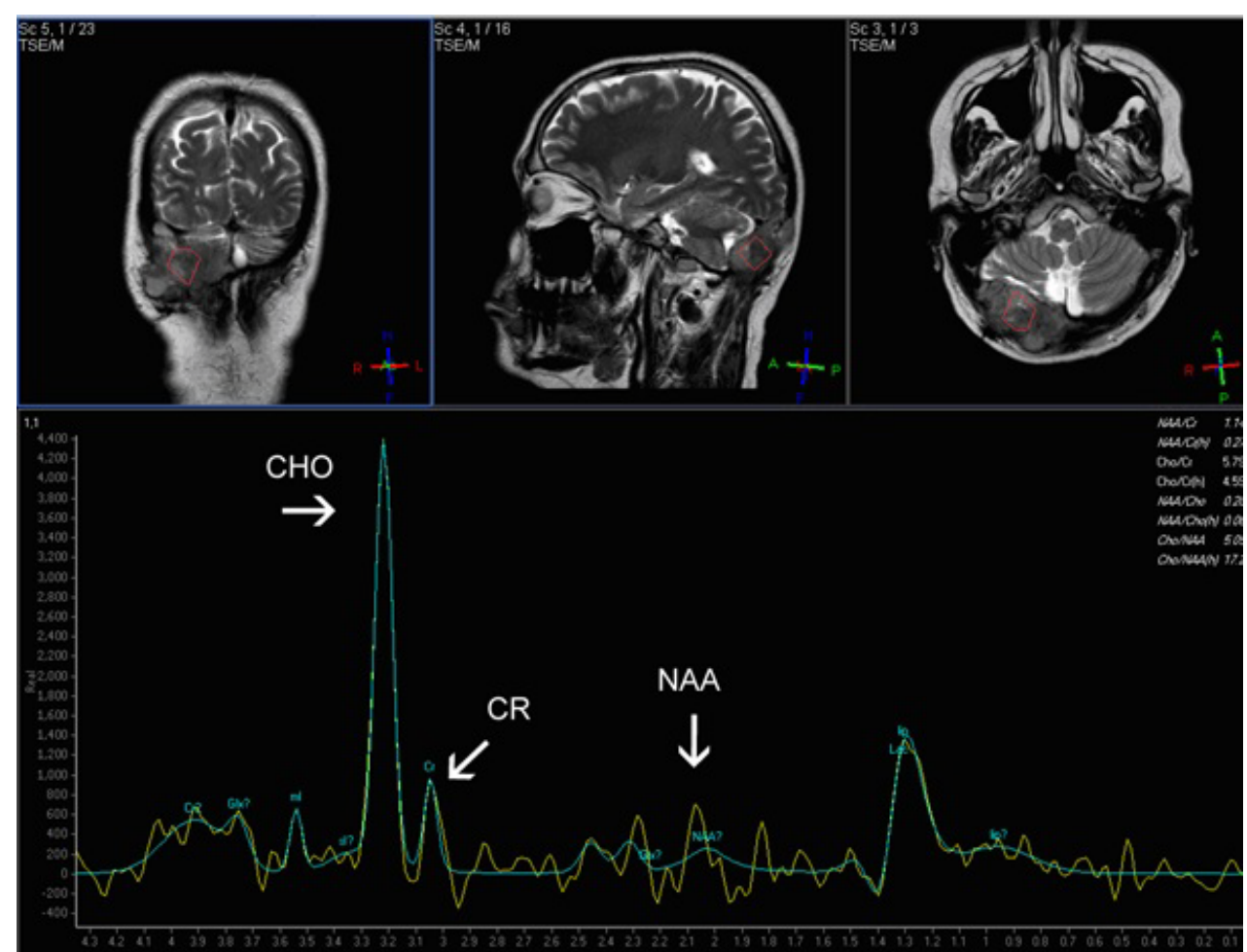

Figure 2: Single voxel H-MRS of the tumor shows elevated choline- $(\mathrm{CHO})$, decreased creatine- (CR) and decreased or absent $\mathrm{N}$-acetylaspartate- (NAA) peaks. 
The tumor could be removed totally with preservation of the transverse sinus patency with negative surgical margins via right sided suboccipital approach. The tumor embraced the right transverse sinus and became ingrown to the bony skull. The cerebellum was compressed but not infiltrated. The dura was intact.

Histological examination revealed a spindle cell tumor with a high cell density and positive reaction for smooth muscle actin (SMA). Diagnosis of leiomyosarcoma was made and based on the FNCLCC classification (Fédération Nationale des Centres de Lutte Contre le Cancer) tumor malignity was scored to grade 2 (Figure 3).

The patient recovered well and was discharged without neurological deficits. Staging examinations with CT of the chest and abdomen revealed no other tumor.

Three months later the patient presented again with a right sided swelling of the neck. MRI of the neck showed contrast enhancing space occupying tissue bordering the former operations field and expanding to caudal. MRI finding was suspicious for recurrence (Figure 4) and hence a new MRS was not done.

The tumor was totally resected and seeds were implanted for adjuvant brachytherapy. The patient received twice a day 2.5 Gy GD 192iridium - HDR - brachytherapy up to 20 Gy. Additionally he received radiotherapy of the corresponding muscle tissue with 50Gy. However final histological examination with reference histology showed scar tissue, without evidence of tumor cells. Three and six months follow up MRI of the brain and spine showed no recurrence.

\section{Discussion}

Bone and soft tissue sarcomas are with a prevalence of 4 in 100000 people per year among rare tumors. LMS occurs more frequent in patients over 55 years ${ }^{5}$. LMS is most

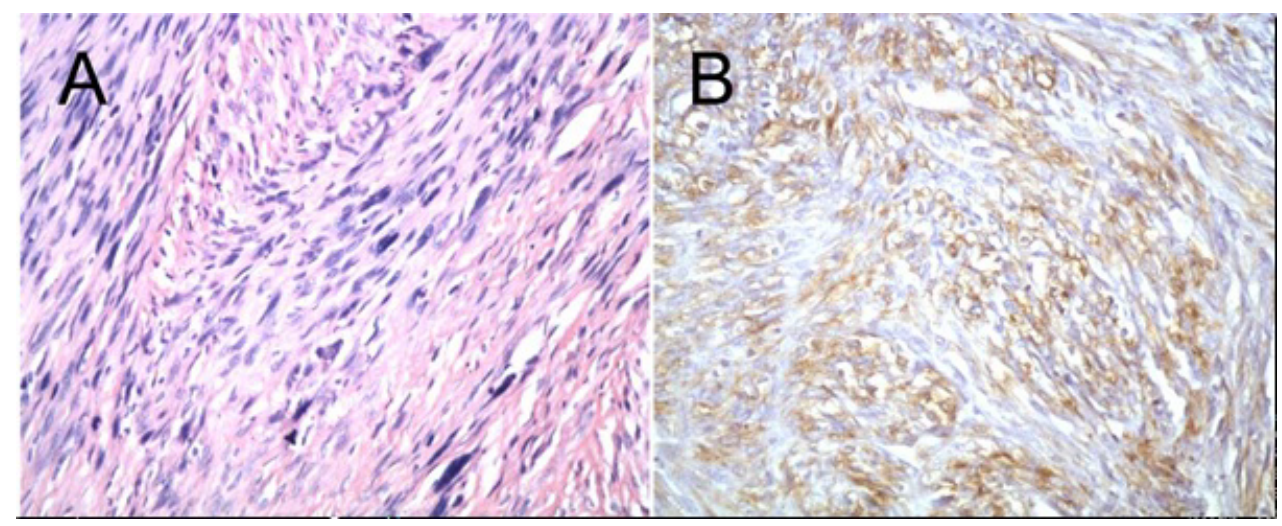

Figure 3: histological preparation of intracranial LMS, A: spindle cell tumor with high cell density (hematoxylin and eosin, maginifaction 20-fokl), B: immunhistochemically reaction with SMA (maginifaction 20-fokl)

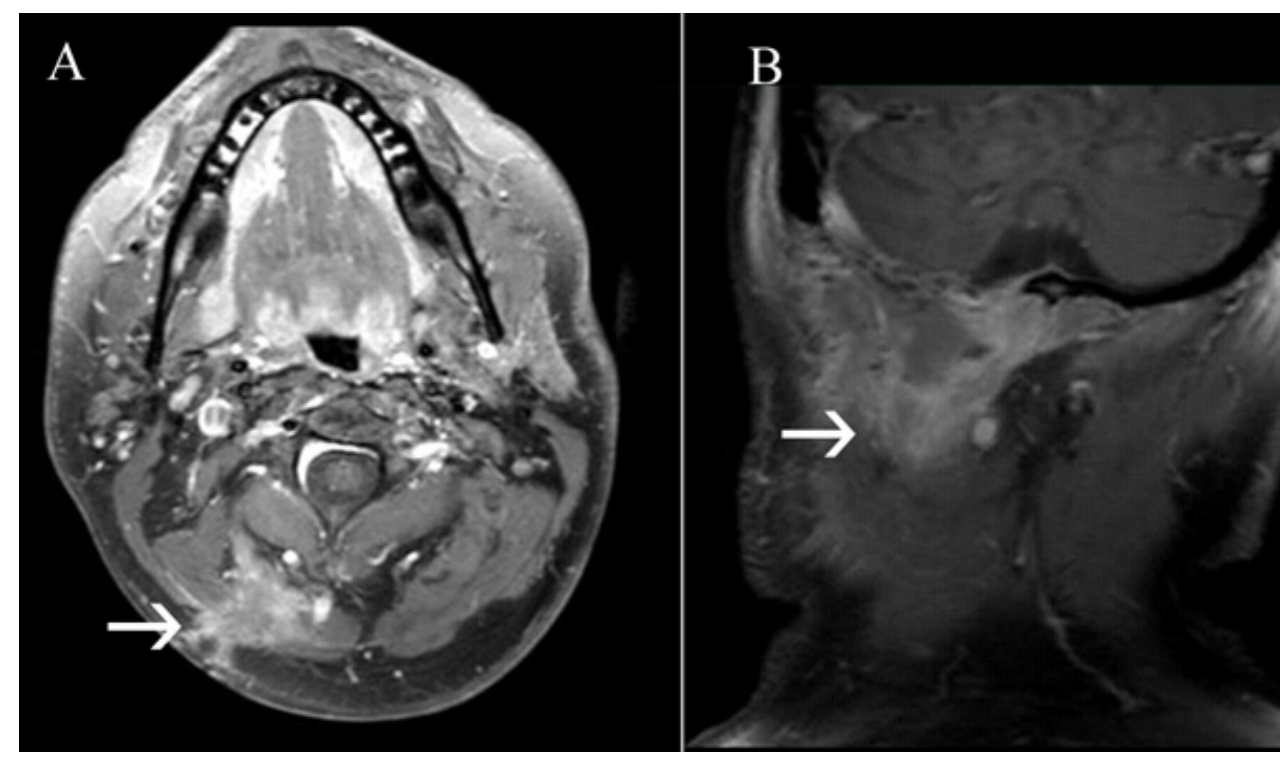

Figure 4: T1 weighted MRI of the neck with contrast in axial (left) and coronar (right) shows swelling in muscle tissue of the neck, suspicious for recurrence tumor 
found in the uterus, stomach, intestines and walls of blood vessels. CNS affection is very infrequent, most consecutive of metastatic disease, whereby metastases usually affect the brain parenchyma ${ }^{6,7}$. For that reason it is important to perform staging examinations via CT or MRI of the chest and abdomen to exclude a possible primary tumor.

Primary LMS of the CNS is extremely rare ${ }^{3}$. Occurrence of LMS of the CNS in association with Ebstein-Barr virus and AIDS is reported and a higher incidence for LMS after radiotherapy has been observed as well ${ }^{8-11}$.

Radiologic diagnosis is difficult to estimate. A cranial CT scan is imprecise in general to differentiate tumor types. Therefore a MRI with contrast should be performed. However, because of frequent dural attachment and contrast enhancement LMS can easily be misdiagnosed as benign tumour e.g. as meningioma.

A MRS is helpful to differentiate between benign and malignant lesions, as we did in our case. This might be useful for decision-making between observant behaviour and surgical treatment.

Another interesting radiological aspect is the possibility of misdiagnosis of tumor recurrence in adjacent soft tissue.

Diagnosis can only be made by histopathological examination. Typical histological findings are spindle cells with a high cell density, positive reaction for SMA and immunoreactivity for desmin.

Neurosurgical treatment should aspire to complete tumor resection. Different studies could demonstrate that total tumor resection correlates positively with the survival time in LMS patients ${ }^{6}$. But it is nearly impossible in intracranial LMS to enable total tumor resection with wide negative margins, like in abdominal tumor localisation. Therefore an adjuvant radio- or chemotherapy needs to be discussed individually.

Pape et al. postulate that soft tissue sarcomas show slow regress tendency during radiotherapy, so that sarcomas seem to be non-sensitive for radiotherapy. But they underline that $40-50 \%$ of soft tissue sarcomas show objective remission after radiotherapy with 60-70 $\mathrm{Gy}^{12}$. Adjuvant brachytherapy show also good results, so that widespread radiation might not be necessary in cases with total tumor resection. For that reason radiotherapy of LMS of the CNS is recommended, even though it might be difficult to obtain cerebral radiotherapy dose. Adjuvant radiotherapy is more substantial in cases, with not complete resectable tumors to prevent regrowth at least.

Study results for chemotherapy on soft tissue sarcomas are heterogeneous. Just few cases are reported with positive response on adjuvant chemotherapy with cyclophosphamide, doxorubicin and DTIC. The usability in overall remains investigational ${ }^{13}$.
Prognosis of soft tissue sarcomas, more specifically LMS, is often poor. Sarcomas tend to early haematogenous spread into different organs.

In a retrospective study on soft tissue sarcomas, patients with brain metastases had a median survival time of 15 months. The most frequent type of sarcomas was LMS. Patients who underwent neurosurgical tumor resection had a much higher survival time in comparison to patients without surgery (2-year post-metastasis survival $27 \%$ vs. $0 \%)^{6}$. There is limited data for survival time of primary intracranial LMS available. Reviewing the literature survival time ranges from 4 to 24 months after neurosurgical treatment.

\section{Conclusion}

Primary LMS of the CNS is very infrequent. Reported cases of intracranial LMS are often metastasic. An elevated incidence in association with Ebstein-Barr virus, AIDS and after radiotherapy is described. If infection is not known, HIV serology could be performed.

MRI is the standard examination for detecting the tumor and its relation to adjacent brain structures. A MRS is helpful to identify malignancy. In view of longest survival time, a complete tumor resection with negative margins is mandatory. Diagnosis can only be verified by histopathological examination. Typical histological findings are spindle cells with a high cell density, positive reaction for smooth muscle actin (SMA) and immunoreactivity for desmin. Once diagnosed LMS, adjuvant radiotherapy is recommended, because $40-50 \%$ of soft tissue sarcomas show remission. Success of adjuvant chemotherapy is currently uncertain. Despite multimodal aggressive therapy, prognosis of LMS remains poor, because of early haematogenous spread.

\section{References}

1. Hussain S, Nanda A, Fowler M, Ampil FL, Burton GV. Primary intracranial leiomyosarcoma: report of a case and review of the literature. - Sarcoma. 2006;2006:52140.

2. Kleihues $P$, Cavenee WK. pathology and genetics of tumors of the nervous system - Lyon : IARC Press; 2000

3. Paulus W, Slowik F, Jellinger K. Primary intracranial sarcomas: histopathological features of 19 cases. Histopathology. 1991;18(5):395402.

4. Sandruck J, Escobar P, Lurain J, Fishman D. Uterine leiomyosarcoma metastatic to the sphenoid sinus: a case report and review of the literature. Gynecol Oncol. 2004;92(2):701-4. Review.

5. Osuna D, de Alava E. Molecular pathology of sarcomas. Rev Recent Clin Trials. 2009;4(1):12-26. Review.

6. Espat NJ, Bilsky M, Lewis JJ, Leung D, Brennan MF. Soft tissue sarcoma brain metastases. Prevalence in a cohort of 3829 patients. Cancer. 2002;94(10):2706-11.

7. Isobe N, Oki S, Sumida M, et al. Metastatic leiomyosarcoma of the brain manifesting as multiple hemorrhages. Case report. Neurol Med Chir (Tokyo). 2005;45(1):44-8. 
8. Lerdlum S, Lalitanantpong S, Numkarunarunrote N, et al. MR imaging of CNS leiomyosarcoma in AIDS patients. J Med Assoc Thai. 2004;87 Suppl 2:S152-60.

9. Suankratay C, Shuangshoti S, Mutirangura A, et al. Epstein-Barr virus infection-associated smooth-muscle tumors in patients with AIDS. Clin Infect Dis. 2005;40(10):1521-8. Epub 2005 Apr 12.

10.Toh CH, Wong HF, Jung SM, Wong AM. Radiation-induced skull base leiomyosarcoma presenting with intracerebral haemorrhage. $\mathrm{Br}$ Radiol. 2007;80(957):e212-5.
11.Yeh DJ, Hessler RB, Lee MR. Leiomyosarcomatous transformation of unknown pediatric primary brainstem lesion following radiotherapy. Case report. Neurosurg Focus. 2002;12(6):ecp2.

12.Pape H, Orth K, Engers R, et al. Radiotherapy of soft tissue sarcoma-part of a multidisciplinary strategy. Wien Klin Wochenschr. 2008;120(23-24):723-31.

13. Yeh DJ, Hessler RB, Lee MR. Leiomyosarcomatous transformation of unknown pediatric primary brainstem lesion following radiotherapy. Case report. Neurosurg Focus. 2002;12(6):ecp2. 